\title{
Esofagitis eosinofílica por sensibilización a proteínas de leche de cabra y oveja
}

\author{
M. Armisén, C. Vidal, L. López-Rosés ${ }^{1}$, V. Rodríguez y B. Bartolomé \\ Servicio de Alergia. Hospital de Conxo. Complejo Hospitalario Universitario de Santiago. Santiago de Compostela. \\ ${ }^{I}$ Servicio de Digestivo. Hospital Xeral-Calde. Lugo. ${ }^{2}$ Laboratorio de Aplicaciones I + D. Bial-Arístegui. Bilbao
}

\section{RESUMEN}

La esofagitis eosinofílica, entidad caracterizada por la infiltración de la mucosa esofágica por más de 20 eosinófilos por campo de gran aumento, se suele presentar en forma de disfagia intermitente de larga evolución, pudiendo estar asociada a sensibilización alérgica a aeroalérgenos y/o alimentos. Presentamos el caso de un varón con clínica de disfagia intermitente coincidiendo con la toma de quesos curados de oveja y cabra que precisó asistencia urgente tras la impactación de un comprimido de ibuprofeno a $30 \mathrm{~cm}$ de la arcada dentaria. El estudio practicado demostró la existencia de estenosis en el esófago a ese nivel con infiltración eosinofílica difusa y sensibilización a proteínas de la leche de cabra, oveja y vaca, con especial relevancia para la IgG bovina, lactoferrina y albúmina sérica. Tras tratamiento con fluticasona deglutida y medidas de evitación se consiguió la resolución del cuadro clínico y la desaparición de los eosinófilos en la mucosa.

Palabras clave: Esofagitis eosinofílica. Disfagia. Alergia. IgG bovina. Lactoferrina. Albúmina bovina sérica.

\begin{abstract}
Eosinophilic esophagitis is an inflammatory disease of the esophagus characterized by the presence of high numbers of eosinophils in the esophageal mucosal layer ( $>20$ high-power field). It is uncommon in adults but in such cases intermittent dysphagia and food impaction are the most common presenting symptoms. We report the case of a male with long-standing intermittent dysphagia after eating selected goat and sheep cheese types, who required medical help following the impaction of an ibuprofen pill in the esophagus. A biopsy demonstrated the presence of eosinophilic inflammation, and allergy testing showed specific IgE against proteins in the milk of goats and sheep. Topical steroid therapy with oral fluticasone, and the elimination of these dairy products from the diet induced complete symptom resolution, and biopsy specimens taken 4 months later showed no eosinophils.
\end{abstract}

Key words: Eosinophilic esophagitis. Dysphagia. Allergy. Bovine IgG. Lactoferrin. Bovine serum albumin.

Armisén M, Vidal C, López-Rosés L, Rodríguez V, Bartolomé B. Esofagitis eosinofílica por sensibilización a proteínas de leche de cabra y oveja. Rev Esp Enferm Dig 2008; 100: 53-56.

\section{INTRODUCCIÓN}

La esofagitis eosinofílica (EE) es una patología inflamatoria de la mucosa del esófago que, aunque poco frecuente, ha suscitado cierto interés en los últimos años por su particular relación con la sensibilización alérgica. $\mathrm{Su}$ diagnóstico pasa por el reconocimiento de infiltración de la mucosa esofágica por eosinófilos (más de 20 eosinófilos por campo de gran aumento), afectando especialmente al tramo proximal y medio del esófago $(1,2)$. Es más

\section{Recibido: 03-08-07.}

Aceptado: 10-09-07.

Correspondencia: Margarita Armistén. Servicio de Alergia. Hospital de Conxo. Rúa Ramón Baltar, s/n. 15706 Santiago de Compostela. e-mail: margarita.armisen.gil@sergas.es frecuente en la edad pediátrica y en pacientes jóvenes, con claro predominio en los varones atópicos (1-3). Su presentación en la edad adulta suele ser en forma de disfagia intermitente de larga evolución, siendo habitual su debut como episodios de impactación alimentaria, si bien puede cursar con vómitos, regurgitación y dolor retroesternal de curso oscilante $(4,5)$. Esta anamnesis se suele asociar a reflujo gastroesofágico y trastornos del peristaltismo, por lo que es importante descartar la presencia del infiltrado eosinofílico.

La detección de sensibilización a aeroalérgenos y alimentos, así como la mejoría clínica observada tras las dietas de exclusión, refuerza el papel de la sensibilización alérgica en la etiopatogenia de la EE (6) y recomienda la realización de minuciosos estudios inmunoalérgicos para tratar de demostrar el papel de cada alérgeno en cada caso. 


\section{CASO CLÍNICO}

Varón de 31 años de edad diagnosticado de hipersensibilidad a proteínas de la leche de vaca en la infancia con tolerancia posterior, rinitis estacional y asma leve intermitente por alergia a polen de gramíneas y al epitelio de gato, por la que había recibido inmunoterapia específica con buena respuesta clínica. Refiere que, en los últimos 4 años y coincidiendo con la toma de quesos curados de oveja y cabra, presenta disfagia alta que cede espontáneamente sin medidas especiales. En enero de 2005 y tras la toma de un comprimido de $400 \mathrm{mg}$ de ibuprofeno, presentó un cuadro agudo de disfagia con clara dificultad para la deglución, incluso, de saliva. Horas después y ante la persistencia del cuadro clínico, acudió al Servicio de Urgencias del Hospital Xeral-Calde, en Lugo, en donde se pudo comprobar la presencia del comprimido impactado a $30 \mathrm{~cm}$ de la arcada dentaria. La endoscopia resultó ser especialmente difícil por la estrechez del esófago a ese nivel, siendo preciso el empleo de un endoscopio ultrafino. El edema de la pared del esófago fue tan marcado que se precisó de la fragmentación del comprimido para su posterior extracción. En ese momento, se tomaron muestras para biopsia en varios puntos del esófago en las que se demostró la presencia del infiltrado eosinofílico (Fig. 1). El paciente fue remitido al Servicio de Alergia en el Complejo Hospitalario Universitario de Santiago en donde se instauró tratamiento con fluticasona deglutida a dosis de $500 \mu \mathrm{g}$ cada 12 horas. Se mantuvo el tratamiento durante 3 meses y se recomendó la abstención de consumo de quesos y productos derivados de leche de cabra y oveja en tanto no se realizase el estudio alergológico. El control endoscópico con sus correspondientes biopsias tomadas 4 meses después no evidenció lesión alguna.

Se realizaron pruebas cutáneas en prick frente a los aeroalérgenos ambientales habituales, incluyendo Dermatophagoides pteronyssinus, Lepidoglyphus destructor, Tyrophagus putrescentiae, Euroglyphus maynei, Lolium perenne, Betula alba, Plantago lanceolata, Parietaria judaica, Blatella germanica, Alternaria alternata, Aspergillus fumigatus, Penicillium notatum, Cladosporium herbarum, látex y epitelios de perro, gato, hámster y vaca, con resultado positivo frente a los pólenes de gramíneas (Lolium perenne) y de Plantago lanceolata, así como a epitelio de gato. Las pruebas cutáneas frente a los extractos comerciales de alimentos, incluyendo almeja, calamar, gamba, mejillón, ostra, leche de vaca, caseína, huevo, trigo, arroz, maíz, lenteja, soja, cacahuete, merluza, sardina, kiwi, nuez y avellana fueron negativas al igual que las pruebas frente a extracto comercial de Anisakis simplex y látex. El paciente aportó unas muestras de queso y yogures de cabra y de oveja con los que se realizó una prueba de prick-prick. La prueba de prick-prick consiste en la realización de una prueba de prick clásica, utilizando una lanceta con la que se hace penetrar el alérgeno en las capas superfi-

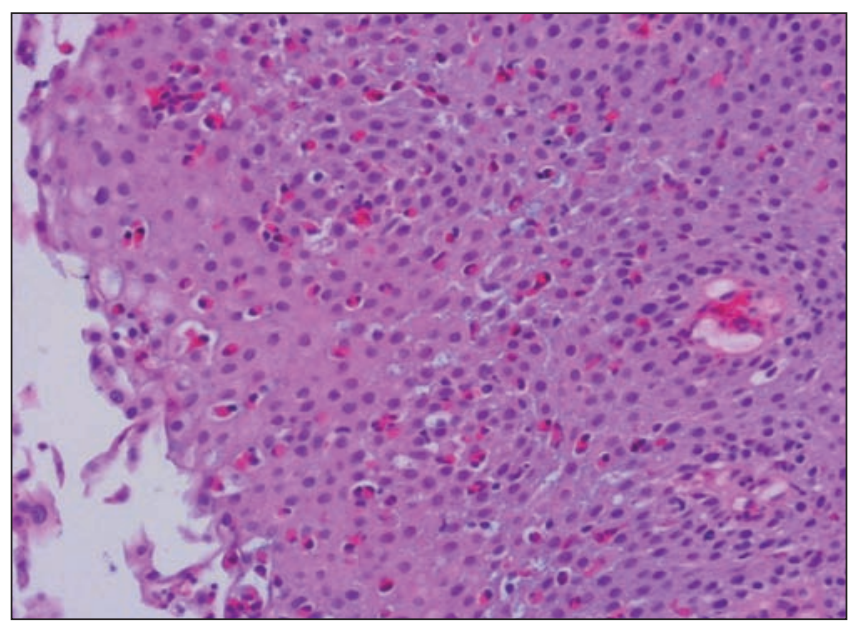

Fig. 1. Detalle de la biopsia de la mucosa esofágica en la que se puede comprobar la presencia de eosinófilos en un número superior a 20 por campo de gran aumento.

ciales de la epidermis en la cara anterior del antebrazo, después de haber puncionado el alimento con dicha lanceta. Con este método, se obtuvo una respuesta positiva frente al queso de cabra (4 x $4 \mathrm{~mm})$, queso de oveja (4 $\mathrm{x}$ $4 \mathrm{~mm})$, yogur de cabra $(9 \times 9 \mathrm{~mm})$ y yogur de oveja (10 $\mathrm{x} 4 \mathrm{~mm}$ ). El control positivo con histamina produjo una pápula de $3 \times 3 \mathrm{~mm}$, considerando positivas aquellas pruebas con un tamaño de pápula igual o superior al obtenido con el control de histamina. Se realizaron pruebas epicutáneas con los quesos de cabra y de oveja para comprobar la posible implicación de mecanismos de hipersensibilidad celular retardada. Para ello se procedió a colocar sendos parches con los citados quesos, permaneciendo ocluidos durante 48 horas pero su resultado fue, en este caso, negativo.

La determinación de la IgE sérica total fue de $422 \mathrm{kU} / 1$ (valores de normalidad por debajo de $100 \mathrm{kU} / \mathrm{l}$ ) y la $\operatorname{IgE}$ específica frente a leche de cabra, oveja y vaca (CAP System $^{\circledast}$, Phadia, Uppsala, Suecia) fue positiva con valores de $0,59,0,43$ y $0,77 \mathrm{kU} / 1$, respectivamente. Mediante la técnica de SDS-PAGE immunoblotting se observaron bandas fijadoras de IgE específica de masa molecular aparente de 85,66 y $55 \mathrm{kDa}$ en los extractos de leche de vaca y leche, yogurt y queso de oveja, cuando los extractos se enfrentaban al suero del paciente. Utilizando esta misma técnica, se pudo identificar en dicho suero IgE específicas que reconocían epítopos presentes en las proteínas lactoferrina, albúmina de suero e inmunoglobulina $\mathrm{G}$ bovinas; las masas moleculares de estas proteínas coinciden con las de las bandas fijadoras de IgE descritas (la cadena pesada de la inmunoglobulina $\mathrm{G}$ bovina tiene una masa molecular de aprox. $56 \mathrm{kDa}$ ) (Fig. 2).

Una vez realizado el diagnóstico y tras la evitación estricta de productos lácteos derivados de oveja y cabra, el paciente ha permanecido asintomático con un control endoscópico posterior normal. 


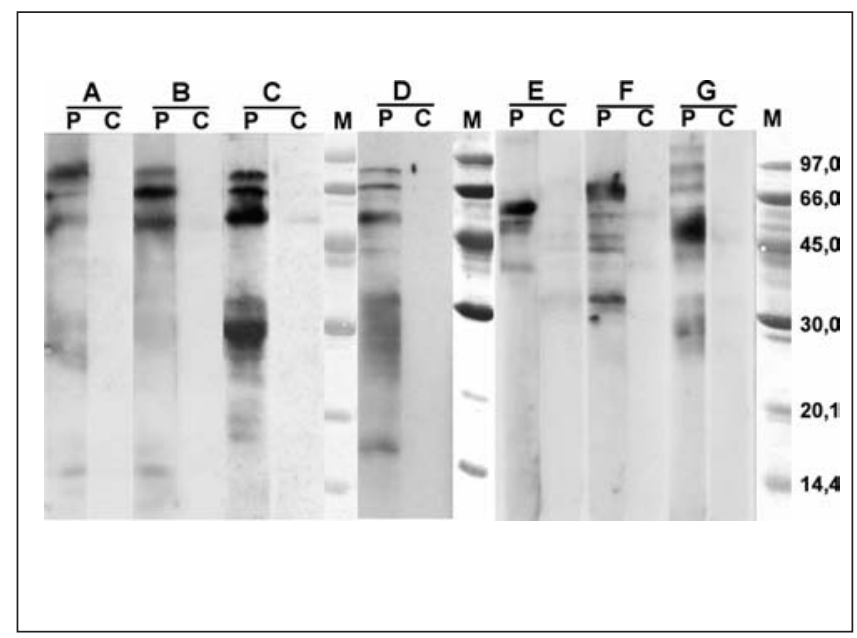

Fig. 2. SDS-PAGE immunoblotting. A. Extracto de leche de vaca; B. Extracto de leche de oveja; $C$. Extracto de yogurt de oveja; D. Extracto de queso de oveja; E. Albúmina del suero bovino; F. Lactoferrina bovina; G. Inmunoglubulina G bovina. Calle P: suero del paciente; calle C: suero control (mezcla de sueros de personas no atópicas); calle M: patrón de masas moleculares.

\section{DISCUSIÓN}

La esofagitis eosinofílica es una patología infrecuente aunque, probablemente, se encuentre infradiagnosticada al requerir de biopsia que confirme la presencia de, al menos, 20 eosinófilos por campo de gran aumento. No existe ninguna alteración macroscópica que se pueda considerar patognomónica e incluso la exploración endoscópica puede ser normal $(2,4)$. Dado que la presencia de eosinofilia en la mucosa esofágica puede aparecer en otros procesos como, por ejemplo, el reflujo gastroesofágico, es preciso confirmar la presencia de dicho infiltrado en diferentes puntos del esófago. Así, en los pacientes con reflujo gastroesofágico importante, es común la presencia de eosinófilos en un número menor a 20 por campo y limitado a la mucosa distal. Por otra parte y aunque el infiltrado eosinofílico suele estar limitado al esófago, se ha descrito la posibilidad de encontrar eosinófilos en zonas más distales como en el antro y el duodeno, pudiendo acompañarse de eosinofilia periférica y constituyendo una forma extensa de enteritis eosinofílica (1).

En los últimos años asistimos a un aumento del número de comunicaciones publicadas a este respecto (3), no sólo en la edad pediátrica sino también en población adulta, que resulta paralelo al incremento de la patología alérgica en general. La frecuente existencia en estos pacientes de antecedentes de atopia, así como la presencia de sensibilización a alimentos y su mejoría tras la realización de dietas de exclusión, pone de manifiesto la implicación de un mecanismo inmunoalérgico $(6,7)$ todavía no suficientemente esclarecido. En algunos casos parece estar implicado un mecanismo de hipersensibilidad inmediato (6-8), mientras que en otros parece intervenir la hi- persensibilidad retardada $(6,7)$. Los resultados obtenidos en el paciente aquí presentado sugieren un mecanismo IgE-mediado y, por tanto, pertenecería al primero de los grupos. Concretamente, el estudio de immunoblot permitió la identificación de bandas correspondientes a proteínas cuya participación en procesos de hipersensibilidad a leche de vaca ha sido destacada en los últimos años por distintos autores $(9,10)$, como la albúmina sérica bovina, la lactoferrina y la inmunoglobulina $\mathrm{G}$ bovina (11), asimismo se detectó fijación en bandas correspondientes a dichas proteínas en la leche de oveja y sus derivados. Por último, destacar que parece que existe cierta susceptibilidad a padecer EE en sujetos portadores de un determinado polimorfismo en el gen de la eotaxina 3 (1), aspecto este que no fue abordado en el paciente.

En relación con la evolución de la EE, a pesar de la buena respuesta clínica del paciente, no es posible predecir su posterior evolución, pues estudios de seguimiento tanto en población pediátrica (12) como en adultos (13) apuntan a la posibilidad de recaídas con independencia de la estrategia terapéutica aplicada. La realización de dietas de exclusión de los alimentos frente a los que se ha detectado una sensibilización consigue la mejoría en más del 75\% de los casos cuando se trata de uno o pocos alimentos (6). Es lógico pensar que en aquellos casos en los que son muchos los alimentos supuestamente implicados o en los que son fruto de reactividades cruzadas con ciertos alérgenos ambientales y en los que la evitación es extremadamente difícil, el fracaso de esta medida sea mayor $(8,14)$. No obstante, incluso en pacientes con múltiples sensibilizaciones, la dieta provoca mejoría cuando se restringe a una dieta elemental (6), siendo su principal limitación el escaso aporte nutricional en este tipo de dieta tan estricta.

En conclusión, el paciente aquí comentado constituye un ejemplo característico de EE: varón, atópico con patología respiratoria previa por aeroalérgenos que refiere historia prolongada e insidiosa de disfagia recurrente. El creciente incremento de la EE debe alertar tanto a los médicos especialistas de alergia como de digestivo, entre los que debe existir una estrecha colaboración que permita un abordaje diagnóstico y terapéutico completo.

\section{BIBLIOGRAFÍA}

1. Norvell JM, Venarske D, Hummell DS. Eosinophilic esophagitis: An allergist's approach. Ann Allergy Asthma Immunol 2007; 98: 207-15.

2. Pasha SF, Dibaise JK, Kim HJ, De Petris G, Crowell MD, Fleischer DE, et al. Patient characteristics, clinical, endoscopic, and histologic findings in adult eosinophilic esophagitis: A case series and systematic review of the medical literature. Dis Esophagus 2007; 20: 311-9.

3. Lucendo Villarín AJ, Carrión Alonso G, Navarro Sánchez M, Martín Chavarri S, Gómez Senent P, Castillo Grau J. Eosinophilic esophagitis in adults, an emerging cause of dysphagia. Description of 9 cases. Rev Esp Enferm Dig 2005; 97: 229-39.

4. Muller S, Puhl S, Vieth M, Stole M. Analysis of symtoms and endoscopic finding in 117 patients with histological diagnoses of eosinophilic esophagitis. Endoscopy 2007; 39: 339-44. 
5. Kerlin P, Jones D, Remedios M, Campbell C. Prevalence of eosinophilic esophagitis in adults with food bolus obstruction of the esophagus. J Clin Gastroenterol 2007; 41: 356-61.

6. Spergel JM, Andrews T, Brown-Whitehorn TF, Beausoleil JL, Liacouras CA. Treatment of eosinophilic esophagitis with specific food elimination diet directed by a combination of skin prick and patch tests. Ann Allergy Asthma Immunol 2005; 95: 336-43.

7. Spergel JM. Eosinophilic esophagitis in adults and children: Evidence for a food allergy component in many patients. Curr Opin Allergy Clin Immunol 2007; 7: 274-8.

8. Plaza-Martín AM, Jiménez-Feijoo R, Andaluz C, Giner-Muñoz MT, Martín Mateos MA, Piquer-Gilbert M, et al. Polysensibilization to aeroallergens and food in eosinophilic esophagitis in a pediatric population. Allergol Immunopathol 2007; 35: 35-7.

9. Jean-Michel Wal. Bovine milk allergenicity (review). Ann Allergy Asthma Immunol 2004; 93: S2-11.
10. Natale M, Bisson C, Monti G, Peltran A, Garoffo LP, Valentini S, et al. Cow's milk allergens identification by two-dimensional immunoblotting and mass spectrometry. Mol Nutr Food Res 2004; 48: 363-9.

11. Ayuso R, Lehrer SB, López M, Reese G, Ibáñez MD, Martín Esteban $\mathrm{M}$, et al. Identification of bovine $\mathrm{IgG}$ as a major cross-reactive vertebrate meat allergen. Allergy 2000: 55: 348-54.

12. Assa'ad AH, Putnam PE, Collins MH, Akers RM, Jameson SC, Kirby CL, et al. Pediatric patients with eosinophilic esophagitis: An 8-year follow-up. J Allergy Clin Immunol 2007: 119: 731-8

13. Straumann A, Spichtin HP, Grize L, Bucher KA, Beglinger C, Simon HU. Natural history of primary esophagitis: A follow-up of 30 adult patients for up to 11.5 years. Gastroenterology 2004; 125: 1660-9.

14. Simon D, Straumann A, Wenk A, Spichtin H, Simon HU, Braathen LR. Eosinophilic esophagitis in adults-no clinical relevance of wheat and rye sensitizations. Allergy 2006: 61: 1480-3. 